\title{
Intramolecular Singlet Fission: Insights from Quantum Dynamical Simulations
}

\author{
S. Rajagopala Reddy, ${ }^{*, \dagger, \S}$ Pedro B. Coto, ${ }^{*, \ddagger}, \|$ and Michael Thoss, $*$ \\ $\dagger$ Institute of Theoretical Physics and Interdisciplinary Center for Molecular Materials, \\ Friedrich-Alexander University Erlangen-Nürnberg, Erlangen, Germany \\ $\ddagger$ Institute of Theoretical Physics, Friedrich-Alexander-University Erlangen-Nürnberg, \\ Erlangen, Germany \\ 【Institute of Physics, Albert-Ludwigs University Freiburg, Freiburg, Germany \\ $\S$ Current address: Department of Computational Sciences, Central University of Punjab, \\ Mansa Road, Bathinda, Punjab 151001, India \\ \|Current address: Department of Physical and Analytical Chemistry, University of Oviedo, \\ Oviedo, Spain
}

E-mail: rajagopala.seelam@fau.de; branapedro@uniovi.es; michael.thoss@physik.uni-freiburg.de 


\begin{abstract}
We investigate the dynamics of intramolecular singlet fission in a dimer consisting of two pentacene-based chromophores covalently bonded to a phenylene spacer using an approach that combines high-level $a b$ initio multireference perturbation theory methods and quantum dynamical simulations. The results show that the population of the multiexcitonic state, corresponding to the first step of singlet fission, is facilitated by the existence of higher-lying doubly excited and charge transfer states that participate in a superexchange-like way. The important role played by high-frequency ring-breathing molecular vibrations in the process is also discussed.
\end{abstract}

\title{
Graphical TOC Entry
}

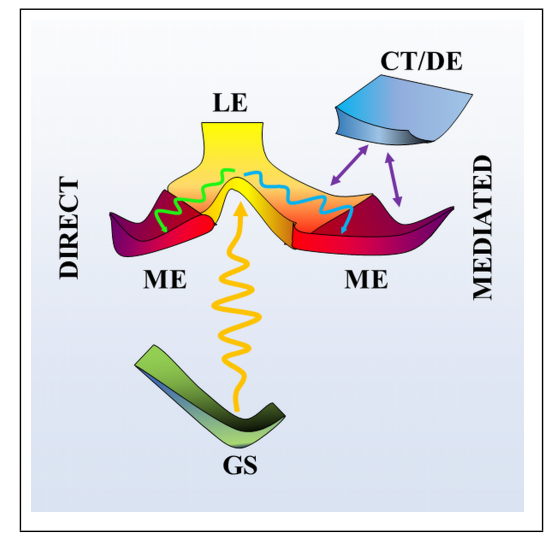


The Shockley-Queisser efficiency limit ${ }^{1}$ imposes a restriction on the maximum performance of single junction solar cells. Over the years, different strategies have been explored to circumvent this limit. One possibility is the use of materials for the energy conversion step exhibiting charge carrier multiplication, ${ }^{2}$ which allows quantum efficiencies larger than $100 \%$ per absorbed photon. It is within this context that singlet fission $(\mathrm{SF})^{3}$ has recently received renewed interest. ${ }^{4,5} \mathrm{SF}$ is a photophysical process in which a chromophore in a singlet electronic excited state $\left(\mathrm{S}_{1}\right)$ shares its excitation energy with another chromophore that is in the ground state and both transform into a pair of triplet electronic excited states $\left(T_{1}\right)$. The nascent $T_{1}$ states are coupled forming a singlet state [also called multiexciton (ME) state], so the process is spin-allowed. ${ }^{6,7}$ Two mechanisms have mainly been advocated to explain SF, the direct and the mediated. ${ }^{3}$ In the direct mechanism, the optically excited state transforms into the ME state, which later dissociates into two (non-interacting) $\mathrm{T}_{1}$ states. In the mediated mechanism, mixing with high-lying charge transfer (CT) states permits the population of the ME state from the optically excited state. Since ionization of the $T_{1}$ states can provide two charge carriers, both mechanisms can potentially lead to the fabrication of highly-efficient SF-based solar cells and initial steps toward this goal have already been taken. ${ }^{8,9}$

Motivated by these technological prospects, the last years have seen important theoretical research efforts devoted to understanding the mechanism of SF at the molecular level. This involves identifying the electronic states participating in the process, their specific roles ${ }^{10-21}$ and coupling mechanisms ${ }^{3,22-26}$ as well as characterizing the dynamics of SF, unraveling the role of coherence, interference, and coupling to molecular vibrations. ${ }^{3,7,14,21,25-31}$ These works have identified key electronic and structural parameters that control the efficiency of the process, a first step in the development of efficient SF-based devices. ${ }^{3,6,29,32-39}$ While most of these works have focused on the investigation of intermolecular SF in organic crystals and films, it has recently become apparent that solutions of covalently linked bichromophoric systems bonded via organic bridges can also undergo SF. ${ }^{40-47}$ This process of intramolecular 
SF (iSF) not only opens the way to solution-processable SF-based photovoltaic devices ${ }^{48}$ but also provides perhaps the best scenario for understanding the mechanism of SF at a molecular level. In this context, a series of 6,13-bis(triisopropylsilylethynyl)pentacene-derived (TIPSP) dimers, where the pentacene-like moieties are covalently bonded to a phenylene spacer in ortho-, meta-, and para-conformations have recently been investigated by some of the authors. ${ }^{43}$ These molecules were designed to impose geometrical restraints and hence control the through-space and through-bond couplings between the electronic states involved in iSF. ${ }^{43}$ Using time resolved spectroscopic techniques and high level ab initio methods, it was shown that mixing between the optically excited state and CT states might be helpful to overcome the activation barrier of SF. Although these results provided insight into the roles of the different excited electronic states involved, the influence of electronic-vibrational coupling, which may be instrumental for efficient SF, ${ }^{49,50}$ was not addressed.

In this Letter, we address this aspect and investigate the dynamics of iSF for a simplified model of $o$-TIPSP, where the triisopropylsilyl groups have been modeled using methyl groups (o-TIPSPm, see Fig. 1). The motivation for the investigation of this particular regioisomer is four-fold. First, this study sheds light on the timescale of SF in this system, an information that could not be obtained in detail from experiment for this system. Second, o-TIPSP has a well defined structure that incorporates both through-space and through-bond couplings and the characterization of the dynamics of SF provides information on vibrational effects on these couplings. Third, CT states appear relatively low in energy in $o$-TIPSP, which allows to ascertain if a significant population of these states promoted by vibronic coupling is possible. Finally, this study provides detailed information on the relative contributions of the direct and mediated channels to SF.

For the simulation of the dynamics of iSF in $o$-TIPSP we have used high-level ab initio multireference perturbation theory methods to build the potential energy surfaces of the different states and the quantum dynamical calculations were carried out using the multilayer multiconfiguration time-dependent Hatree (ML-MCTDH) method. ${ }^{51-58}$ Specifically, we solve 


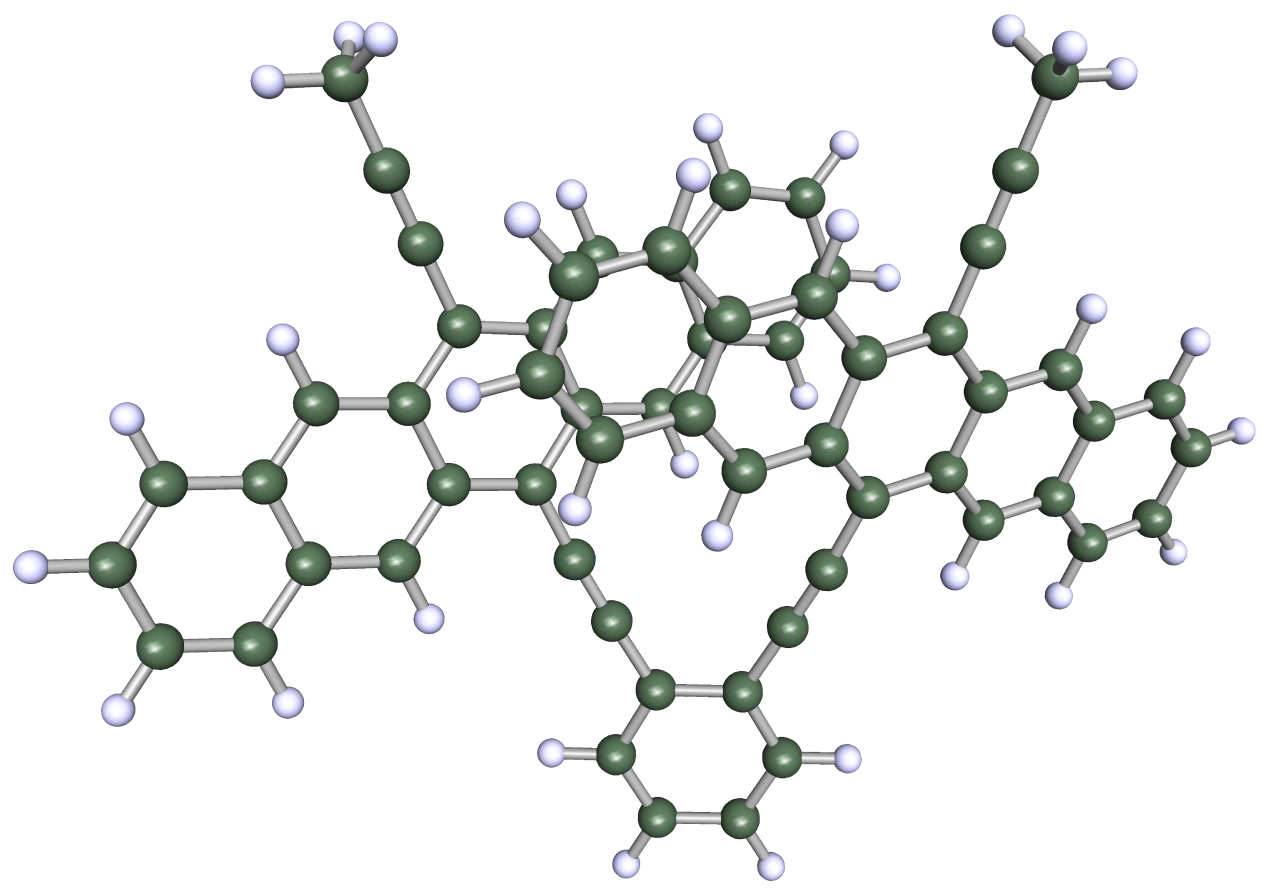

Figure 1: o-Bis(13-(methylethynyl)pentacen-6-yl)ethynyl)benzene dimer (o-TIPSPm) investigated in this work.

the time-dependent Schrödinger equation using a vibronic coupling model Hamiltonian ${ }^{59}$ represented in a basis of diabatic states relevant for the process (see below). This model assumes that the initial state is prepared by an instantaneous excitation to the bright state (i.e. consistent with an experiment where an ultrashort femtosecond laser pulse is used). In the diabatic basis, the Hamiltonian reads $(\hbar=1)$

$$
H=\sum_{I} H_{I}|I\rangle\langle I|+\sum_{I>J}\left(V_{I, J}|I\rangle\langle J|+\text { h.c. }\right)
$$

where $I$ and $J$ label the different diabatic electronic states. In this expression, the diagonal and off-diagonal terms take the form

$$
H_{I}=E_{I}^{0}+\sum_{l} \frac{\omega_{l}}{2}\left(Q_{l}^{2}+P_{l}^{2}\right)+\sum_{l}\left(\kappa_{I, I}^{l} Q_{l}+\frac{1}{2} \gamma_{I, I}^{l} Q_{l}^{2}\right)
$$


and

$$
V_{I, J}=V_{I, J}^{0}+\sum_{l}\left(\kappa_{I, J}^{l} Q_{l}+\frac{1}{2} \gamma_{I, J}^{l} Q_{l}^{2}\right)
$$

Thereby, $E_{I}^{0}$ and $V_{I, J}^{0}$ are the diagonal and off-diagonal elements of the electronic Hamiltonian $\left(H_{\mathrm{el}}\right)$, i.e., the energies and electronic couplings of the diabatic electronic states considered in the model calculated at the ground state reference geometry. $Q_{l}$ and $P_{l}$ are the (dimensionless) coordinates and momenta of the vibrational degrees of freedom, represented by the normal modes in the electronic ground state of $o$-TIPSm. The term $\sum_{l} \frac{\omega_{l}}{2}\left(Q_{l}^{2}+P_{l}^{2}\right)$ describes the kinetic and potential energy corresponding to these vibrational normal modes within the harmonic approximation. The other terms account for the first and second order vibronic couplings, with $\kappa_{I, J}^{l}$ and $\gamma_{I, J}^{l}$ being the linear and quadratic coupling constants for mode $l$, respectively. In the calculations reported below, 28 vibrational were included, which were selected based on the value of their respective dimensionless coupling strengths $\left(\frac{\kappa^{2}}{2 \omega^{2}}\right.$, see Supporting Information). ${ }^{59}$

Based on the model Hamiltonian, Eq. (1), we consider different observables to analyze distinct aspects of the SF process. The electronic dynamics is described by the time-dependent population of the different states involved in the process, given by

$$
P_{L}(t)=\operatorname{Tr}\left\{P_{L} \rho(t)\right\}
$$

where $P_{L}=|L\rangle\langle L|$ is the projector onto the diabatic electronic state $|L\rangle$ and $\rho(t)=$ $e^{-i H t} \rho(0) e^{i H t}$ is the density matrix at time $t$. The initial density matrix is given by

$$
\rho(0)=|I\rangle\left|0_{0}\right\rangle\left\langle 0_{0}\right|\langle I|
$$

where $|I\rangle$ is the initially excited electronic states and $\left|0_{0}\right\rangle$ is the vibrational ground state in the electronic ground state of $o$-TIPSm (see Supporting Information). Thereby, we have 
assumed an ultrafast (instanteneous) excitation of the optically bright state $|L\rangle$ and neglected temperature effects in the initial state. In addition, the autocorrelation function of the inititally excited state is considered,

$$
C(t)=\left\langle 0_{0}\left|\left\langle I\left|e^{-i H t}\right| I\right\rangle\right| 0_{0}\right\rangle
$$

the Fourier transform of which provides the photoabsorption cross-section.

The parameters of the vibronic Hamiltonian, including the diabatic electronic states, energies and coupling constants, were obtained from the corresponding adiabatic electronic states using the diabatization method of Nakamura and Truhlar. ${ }^{60,61}$ The adiabatic electronic states were calculated using the extended multiconfigurational quasidegenerate perturbation theory method (XMCQDPT) ${ }^{62}$ a double- $\zeta$ (DZV) basis set ${ }^{63}$ and employing an eight rootsequal weights state average complete active space self-consistent field (CASSCF) calculation as reference. An active space of four electrons in four orbitals (HOMO and LUMO of each pentacene-like moiety) was used (see Supporting Information for further details).

Table 1 contains the adiabatic vertical excitation energy, modulus of the dipole moment, oscillator strength and character of the lowest lying singlet excited states of $o$-TIPSPm calculated at the ground state equilibrium geometry. The four lowest-lying singlet excited states exhibit ME $\left(\mathrm{S}_{1}\right)$, locally excited (LE)-like $\left(\mathrm{S}_{2}\right.$ and $\left.\mathrm{S}_{3}\right)$, and CT $\left(\mathrm{S}_{4}\right)$ character. These states lie in a close range of energy $(\sim 0.2 \mathrm{eV}$, see Table 1$)$, with $\mathrm{S}_{2}$ and $\mathrm{S}_{3}$ being quasidegenerate. In addition, $\mathrm{S}_{4}$ is also near degenerate with $\mathrm{S}_{5}$, a doubly excited (DE) state. As a result of the small energy differences, there is a significant degree of mixing of the CT state with the rest of states, in particular with the LE and ME states. Finally, the last two states $\mathrm{S}_{6}$ and $\mathrm{S}_{7}$ of DE and CT character, respectively, are quasidegenerate and lie $\sim 0.2 \mathrm{eV}$ above $\mathrm{S}_{5}$.

We have used these adiabatic states to build the diabatic basis employed for the simulation of the dynamics of iSF. This basis comprises the electronic ground state $\left|{ }^{1}\left(\mathrm{~S}_{0} \mathrm{~S}_{0}\right)\right\rangle$, the LE 
Table 1: Adiabatic vertical excitation energy $(\Delta \mathrm{E}$, in $\mathrm{eV}),{ }^{a}$ modulus of the dipole moment $\left(\mu\right.$, in D),$^{b}$ oscillator strength $(f)^{b}$ and character (char.) ${ }^{c}$ of the lowestlying singlet excited states of $o$-TIPSPm calculated at the ground state equilibrium structure.

\begin{tabular}{cccrc}
\hline State & $\Delta E$ & $\mu$ & \multicolumn{1}{c}{$f$} & char. \\
\hline $\mathrm{S}_{1}$ & 1.51 & 2.04 & $<0.001$ & $\mathrm{ME}$ \\
$\mathrm{S}_{2}$ & 1.75 & 2.48 & 1.003 & $\mathrm{LE}$ \\
$\mathrm{S}_{3}$ & 1.78 & 3.24 & 0.270 & $\mathrm{LE}$ \\
$\mathrm{S}_{4}$ & 1.98 & 3.32 & 0.104 & $\mathrm{CT}$ \\
$\mathrm{S}_{5}$ & 2.00 & 2.48 & $<0.001$ & $\mathrm{DE}$ \\
$\mathrm{S}_{6}$ & 2.20 & 1.54 & 0.001 & $\mathrm{DE}$ \\
$\mathrm{S}_{7}$ & 2.25 & 1.63 & 0.002 & $\mathrm{CT}$ \\
\hline
\end{tabular}

${ }^{a}$ Calculated at the XMCQDPT/DZV level of theory ( 8 roots with equal weights and a 4 electron in 4 orbitals active space were used in the CASSCF calculation). ${ }^{b}$ Calculated at the CASSCF/DZV level of theory ( 8 roots with equal weights and a 4 electron in 4 orbitals active space were used). ${ }^{c} \mathrm{ME}=$ multiexcitonic state, $\mathrm{LE}=$ optically bright states that correlate with the plus and minus combinations of locally excited states of both pentacene monomers, $\mathrm{CT}=$ charge transfer states, $\mathrm{DE}=$ doubly excited states.

Table 2: Diabatic electronic Hamiltonian $\left(H_{\mathrm{el}}\right)$ showing the energies and coupling matrix elements $(\mathrm{meV})$ of the low-lying diabatic electronic states used in the simulation of the dynamics of the iSF process of $o$-TIPSPm calculated at the ground state equilibrium structure. ${ }^{a}$

\begin{tabular}{c|rrrrrrrr}
\hline$H_{\mathrm{el}}$ & $\left|{ }^{1}\left(\mathrm{~S}_{0} \mathrm{~S}_{0}\right)\right\rangle$ & $\left.\left.\right|^{1}\left(\mathrm{~S}_{1} \mathrm{~S}_{0}\right)\right\rangle$ & $\left|{ }^{1}\left(\mathrm{~S}_{0} \mathrm{~S}_{1}\right)\right\rangle$ & $\left|{ }^{1}\left(\mathrm{~T}_{1} \mathrm{~T}_{1}\right)\right\rangle$ & $\left|{ }^{1}(\mathrm{CA})\right\rangle$ & $\left|{ }^{1}(\mathrm{AC})\right\rangle$ & $\left|{ }^{1}(\mathrm{DE})_{1}\right\rangle$ & $\left|{ }^{1}(\mathrm{DE})_{2}\right\rangle$ \\
\hline$\left\langle{ }^{1}\left(\mathrm{~S}_{0} \mathrm{~S}_{0}\right)\right|$ & 0 & 15 & -15 & -18 & -108 & 107 & -426 & 416 \\
$\left\langle{ }^{1}\left(\mathrm{~S}_{1} \mathrm{~S}_{0}\right)\right|$ & 15 & 1576 & 23 & 7 & -30 & -43 & 52 & -7 \\
$\left\langle{ }^{1}\left(\mathrm{~S}_{0} \mathrm{~S}_{1}\right)\right|$ & -15 & 23 & 1577 & -7 & -44 & -31 & -9 & 51 \\
$\left\langle{ }^{1}\left(\mathrm{~T}_{1} \mathrm{~T}_{1}\right)\right|$ & -18 & 7 & -7 & 1595 & -273 & 273 & -26 & 24 \\
$\left\langle{ }^{1}(\mathrm{CA})\right|$ & -108 & -30 & -44 & -273 & 1744 & 26 & 43 & -36 \\
$\left\langle{ }^{1}(\mathrm{AC})\right|$ & 107 & -43 & -31 & 273 & 26 & 1744 & -39 & 42 \\
$\left\langle{ }^{1}(\mathrm{DE})_{1}\right|$ & -426 & 52 & -9 & -26 & 43 & -39 & 1809 & 176 \\
$\left\langle{ }^{1}(\mathrm{DE})_{2}\right|$ & 416 & -7 & 51 & 24 & -36 & 42 & 176 & 1821 \\
\hline
\end{tabular}

${ }^{a}$ Calculated at the XMCQDPT/DZV level of theory (8 roots with equal weights and a 4 electron in 4 orbitals active space were used in the CASSCF calculation).

states $\left.\left.\right|^{1}\left(\mathrm{~S}_{0} \mathrm{~S}_{1}\right)\right\rangle$ and $\left.\left.\right|^{1}\left(\mathrm{~S}_{1} \mathrm{~S}_{0}\right)\right\rangle$ with the excitation $\left(\mathrm{S}_{1}\right)$ localized in one pentacene moiety, the ME state $\left.\left.\right|^{1}\left(\mathrm{~T}_{1} \mathrm{~T}_{1}\right)\right\rangle$ (also called correlated triplet pair state), and the CT states $\left.\left.\right|^{1}(\mathrm{CA})\right\rangle$ and $\left.\left.\right|^{1}(\mathrm{AC})\right\rangle$, where $\mathrm{C}$ and $\mathrm{A}$ stand for the radical cation and radical anion form of the pentacene moiety. Due to energy considerations, two doubly excited (DE) diabatic states $\left.\left.\right|^{1}(\mathrm{DE})_{1}\right\rangle$ 
and $\left|{ }^{1}(\mathrm{DE})_{2}\right\rangle$ were also included. Table 2 depicts the diabatic electronic Hamiltonian $\left(H_{\mathrm{el}}\right)$ calculated at the ground state equilibrium geometry of $o$-TIPSPm. The results show that the direct electronic couplings between the ME state and the LE states are non-negligible but small. In contrast, the electronic couplings of the ME and LE states and the CT states are larger, in particular those of the ME state. The electronic couplings of the DE states to the ME and LE states exhibit a more complex behaviour. The couplings of these states to the ME state are moderate and of the same order of magnitude. In contrast, each DE state shows couplings to the LE states that differ by nearly an order of magnitude (see Table 2).

In order to assess the reliability of the vibronic model Hamiltonian used, we have first considered the absorption spectrum. Fig. 2 depicts a comparison of the experimental ${ }^{43}$ and the simulated absorption spectra, the latter calculated using the Fourier transform of the time-autocorrelation function, see Eq. (5). The spectra have similar shapes in the range of energies considered, exhibiting a vibrational progression like that of the absorption spectrum of pentacene where the main vibrational modes involved are $\nu_{19}, \nu_{25}$, and $\nu_{27}$ (see Supporting Information). Interestingly, both show a very weak absorption feature in the region around $\sim 1.6 \mathrm{eV}$. This weak absorption is caused by intensity borrowing from $\left|{ }^{1}\left(\mathrm{~S}_{1} \mathrm{~S}_{0}\right)\right\rangle$ to $\left|{ }^{1}\left(\mathrm{~T}_{1} \mathrm{~T}_{1}\right)\right\rangle$ facilitated by the CT states, ${ }^{21}$ which have energy gaps with respect to the LE and ME states commensurate with the energies of the high-frequency vibrational normal modes included in the simulation (see Supporting Information). ${ }^{31}$

Employing the model introduced above, we have simulated the vibronic dynamics of $o$ TIPSPm after instantaneous population of $\left.\left.\right|^{1}\left(\mathrm{~S}_{1} \mathrm{~S}_{0}\right)\right\rangle$ via photoexcitation. The time evolution of the population of the different diabatic states considered in the model is depicted in Fig. 3. The short time dynamics until $\sim 20$ fs is characterized by the transfer of $\sim 70 \%$ population from $\left|{ }^{1}\left(\mathrm{~S}_{1} \mathrm{~S}_{0}\right)\right\rangle$ mainly to $\left.\left.\right|^{1}(\mathrm{DE})_{1}\right\rangle(\sim 30 \%)$ and $\left|{ }^{1}\left(\mathrm{~S}_{0} \mathrm{~S}_{0}\right)\right\rangle(\sim 20 \%)$, with smaller population transfer $(\sim 10 \%)$ to both $\left|{ }^{1}\left(\mathrm{~T}_{1} \mathrm{~T}_{1}\right)\right\rangle$ and $\left|{ }^{1}\left(\mathrm{~S}_{0} \mathrm{~S}_{1}\right)\right\rangle$. From 20 fs to $50 \mathrm{fs}$, the population of both $\left|{ }^{1}\left(\mathrm{~T}_{1} \mathrm{~T}_{1}\right)\right\rangle$ and $\left|{ }^{1}\left(\mathrm{~S}_{0} \mathrm{~S}_{1}\right)\right\rangle$ rises, reaching values of $\sim 30 \%$ at $50 \mathrm{fs}$. For longer times, the $\left.\left.\right|^{1}\left(\mathrm{~T}_{1} \mathrm{~T}_{1}\right)\right\rangle$ state is increasingly populated, reaching a value of $\sim 70 \%$ at $300 \mathrm{fs}$, while the rest 


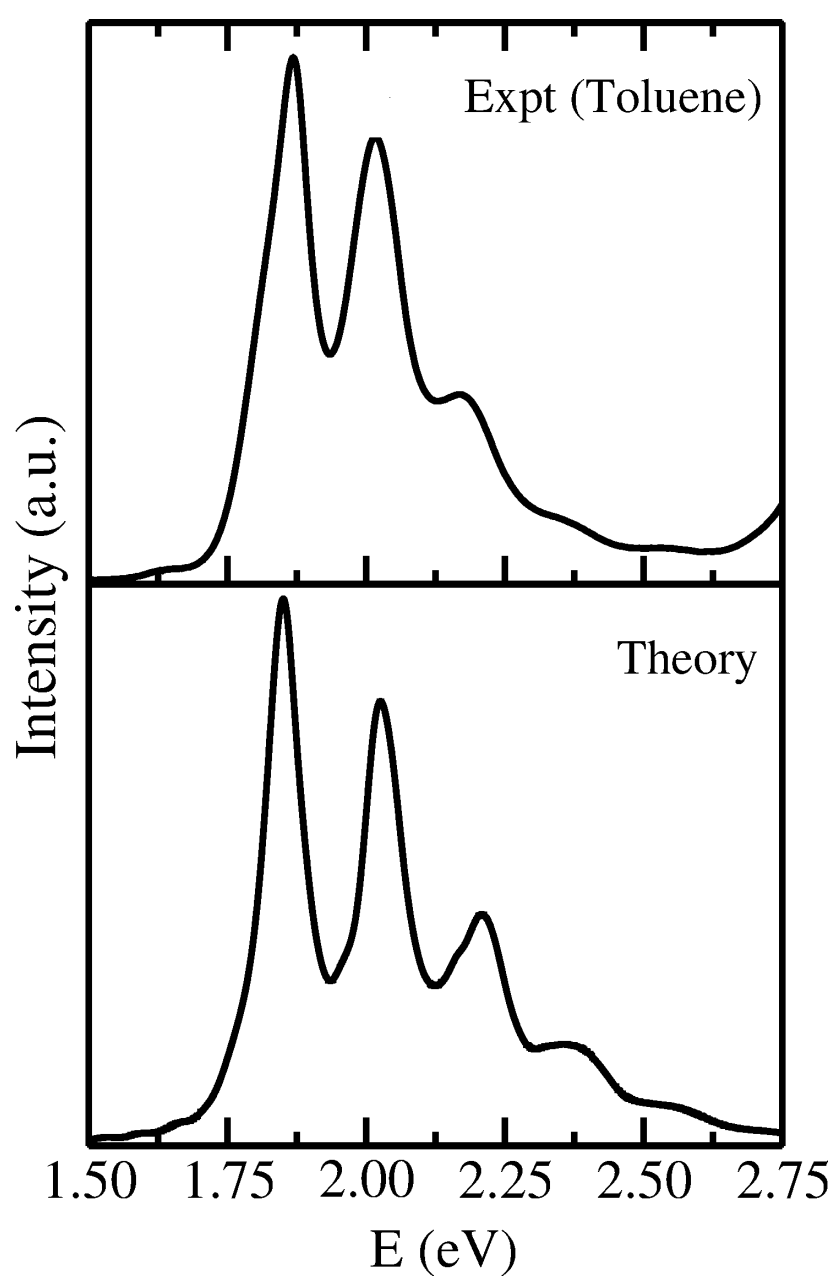

Figure 2: Experimental (in toluene) ${ }^{43}$ (a) and simulated (b) absorption spectra of $o$-TIPSPm in the range $1.50 \mathrm{eV}$ to $2.75 \mathrm{eV}$. In the simulated spectrum, the time-autocorrelation function obtained from the wavepacket propagation calculations has been damped with an exponential function ( $e^{-\frac{t}{\tau}}$, with $\tau=33 \mathrm{fs}$ ) and Fourier transformed to generate the spectral envelope.

of states show populations in the range between $5 \%$ to $10 \%$. Interestingly, after 150 fs the population of $\left.\left.\right|^{1}\left(T_{1} T_{1}\right)\right\rangle$ exhibits small oscillations that are mimicked to a major extent by those shown by the CT states.

These dynamical results predict that the first step of iSF in $o$-TIPSPm proceeds on an ultrafast timescale of $\sim 100 \mathrm{fs}$. This is in accordance with previous experimental work employing time-resolved spectroscopic techniques, which gave an upper limit of 500 fs for the population of $\left|{ }^{1}\left(\mathrm{~T}_{1} \mathrm{~T}_{1}\right)\right\rangle .{ }^{43}$

Regarding the mechanism of iSF in $o$-TIPSPm, it is important to note that the dynamical 


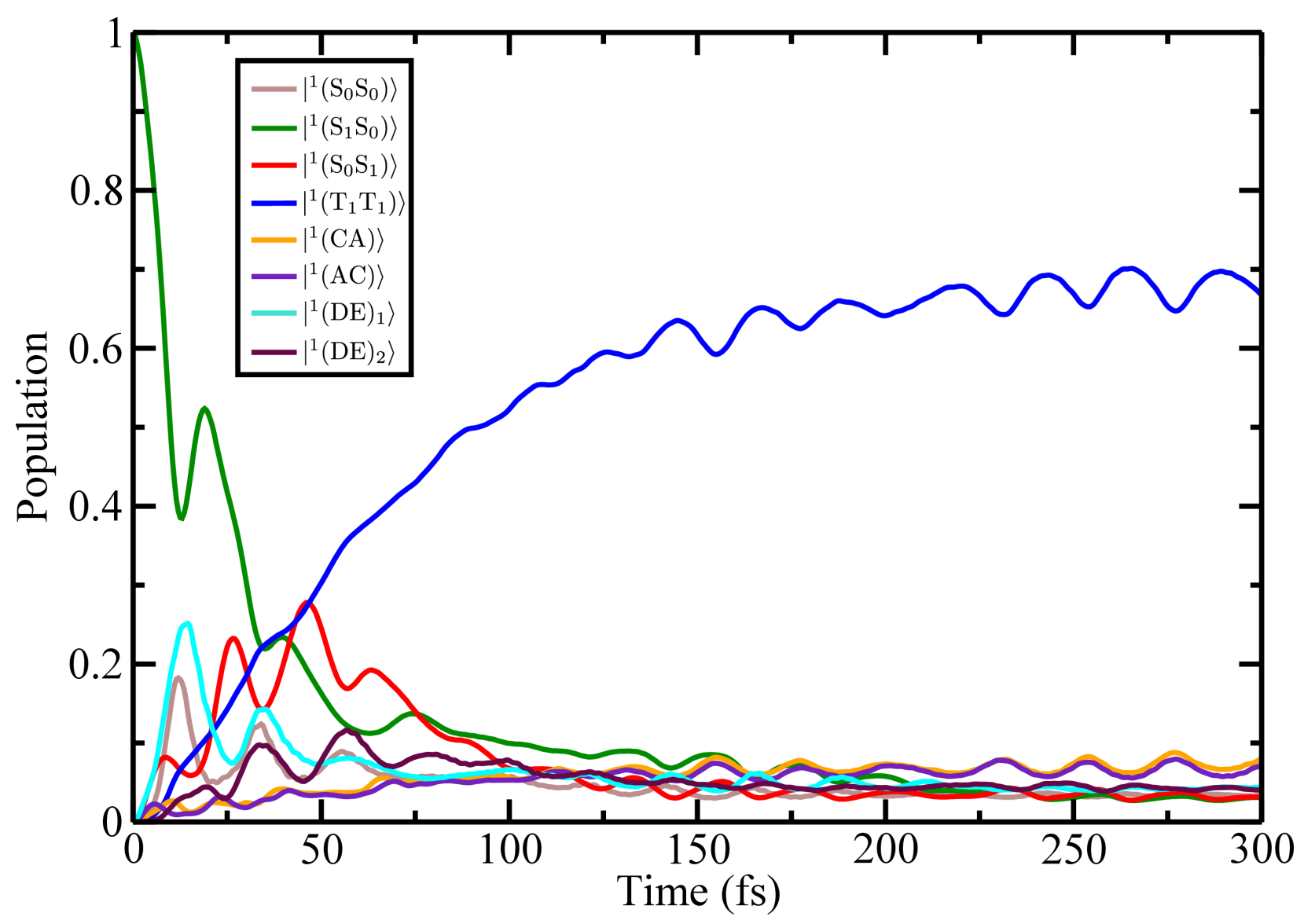

Figure 3: Time evolution of the population of the diabatic states involved in the iSF process of $o$-TIPSPm after instantaneous photoexcitation of $\left|{ }^{1}\left(\mathrm{~S}_{1} \mathrm{~S}_{0}\right)\right\rangle$.

results for the full vibronic model, depicted in Fig. 3, are very different from those obtained for the purely electronic dynamics (i.e. using a model Hamiltonian without coupling to vibrations). Here the main features observed are Rabi-like population oscillations between $\left|{ }^{1}\left(\mathrm{~S}_{1} \mathrm{~S}_{0}\right)\right\rangle$ and $\left.\left.\right|^{1}\left(\mathrm{~S}_{0} \mathrm{~S}_{1}\right)\right\rangle$ with negligible population of $\left.\left.\right|^{1}\left(\mathrm{~T}_{1} \mathrm{~T}_{1}\right)\right\rangle$ (see Fig. $\mathrm{S} 4$ in Supporting Information). Thus, the ultrafast iSF process in $o$-TIPSPm is facilitated by strong vibronic coupling.

To further investigate the mechanism of iSF in $o$-TIPSPm, we have analyzed the contributions of the direct and mediated channels to the process. To this end, we have simulated the dynamics of iSF using reduced vibronic model Hamiltonians that differ from the complete model of Eqs. (1)-(3) either in the diabatic basis sets used or in the vibrational normal modes considered (see Supporting Information), obtaining in this manner insight into the 

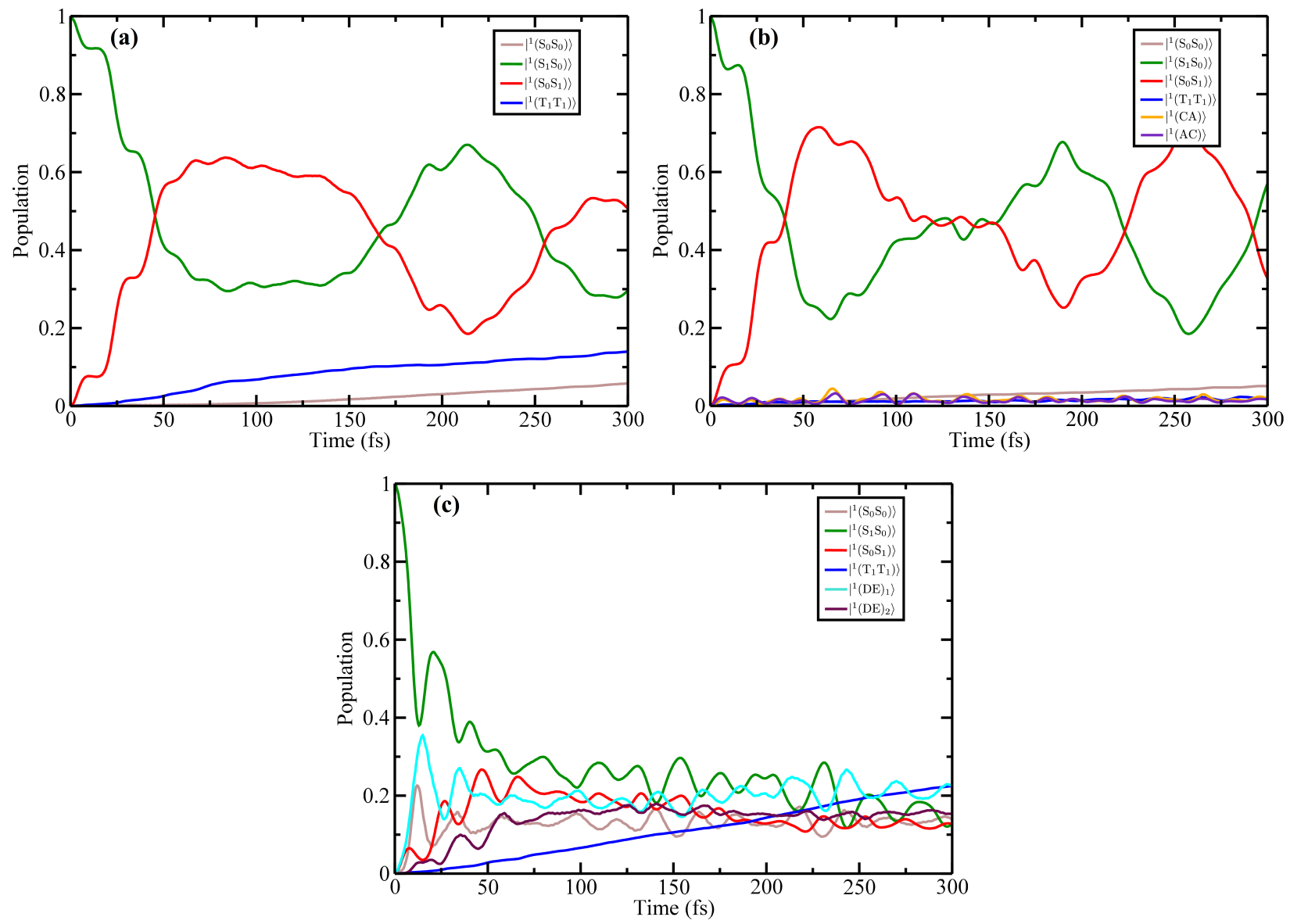

Figure 4: Simulation of the dynamics of the iSF process of $o$-TIPSPm using vibronic model Hamiltonians represented in reduced diabatic basis sets ((a)-(c), see insets). The time evolution of the population of the different diabatic states after instantaneous photoexcitation of $\left.\left.\right|^{1}\left(\mathrm{~S}_{1} \mathrm{~S}_{0}\right)\right\rangle$ is shown.

roles of higher-lying diabatic states (e.g., CT and DE states) and molecular vibrations in the process. Fig. 4 (a) depicts the time evolution of the population of $\left.\left.\right|^{1}\left(T_{1} T_{1}\right)\right\rangle$ for a model Hamiltonian represented in the basis consisting of only the four lowest-lying diabatic states, namely $\left.\left.\left|{ }^{1}\left(\mathrm{~S}_{0} \mathrm{~S}_{0}\right)\right\rangle,\left.\right|^{1}\left(\mathrm{~T}_{1} \mathrm{~T}_{1}\right)\right\rangle,\left.\right|^{1}\left(\mathrm{~S}_{1} \mathrm{~S}_{0}\right)\right\rangle$, and $\left.\left.\right|^{1}\left(\mathrm{~S}_{0} \mathrm{~S}_{1}\right)\right\rangle$ (see Supporting Information for details). Removal of CT and DE states from the vibronic model Hamiltonian has a drastic impact on the iSF dynamics, which features primarily population oscillations between $\left.\left.\right|^{1}\left(\mathrm{~S}_{1} \mathrm{~S}_{0}\right)\right\rangle$ and $\left.\left.\right|^{1}\left(\mathrm{~S}_{0} \mathrm{~S}_{1}\right)\right\rangle$ and a pronounced reduction of the population transfer to $\left.\left.\right|^{1}\left(\mathrm{~T}_{1} \mathrm{~T}_{1}\right)\right\rangle$ that reaches a value of only $\sim 15 \%$ at $300 \mathrm{fs}$. This result indicates that the direct channel plays a minor role in the mechanism of iSF in $o$-TIPSPm. Addition of the two CT states to this reduced 

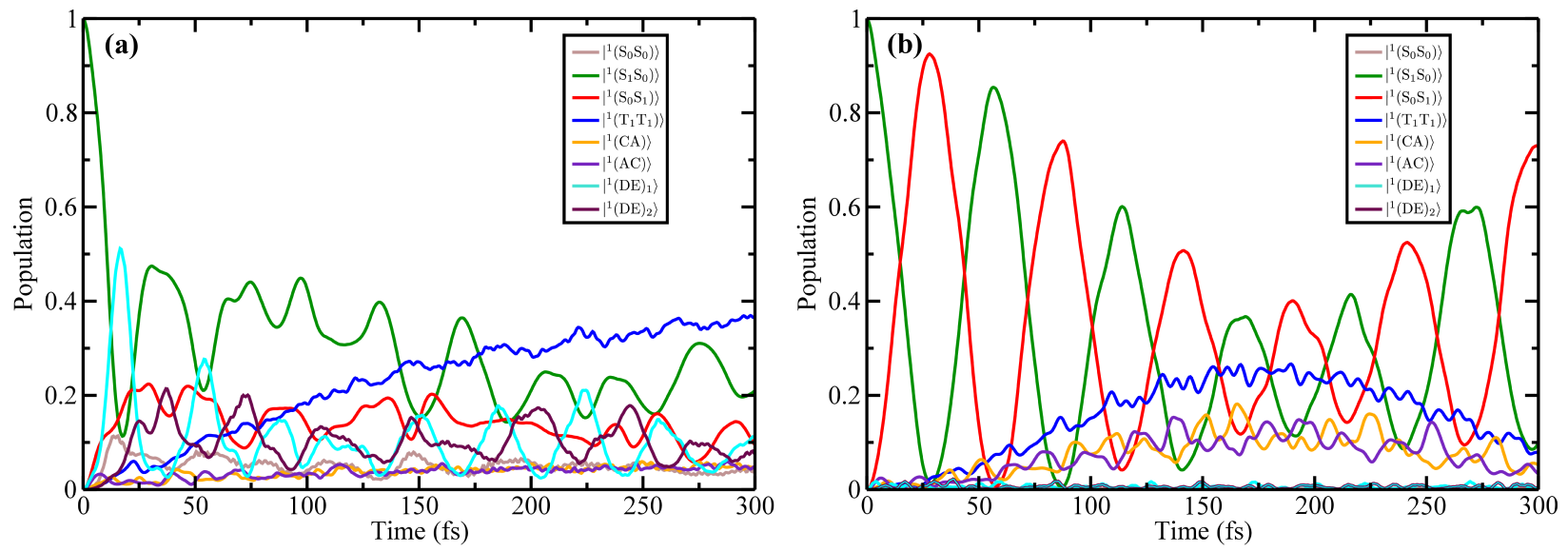

Figure 5: Time evolution of the population of the diabatic states involved in the iSF process of $o$-TIPSPm after instantaneous photoexcitation of $\left|{ }^{1}\left(\mathrm{~S}_{1} \mathrm{~S}_{0}\right)\right\rangle$ obtained using a vibronic model Hamiltonian that includes (a) a subset of 8 vibrational modes most relevant for the iSF process and (b) the ring-breathing vibrational mode with frequency $1350 \mathrm{~cm}^{-1}(\mathrm{~b})$.

model Hamiltonian (see Fig. 4 (b)) results in an even smaller population of $\left.\left.\right|^{1}\left(\mathrm{~T}_{1} \mathrm{~T}_{1}\right)\right\rangle$. This somewhat counterintuitive finding is a result of the opposite signs of the different coupling contributions involving the CT states (see Table 2 and Supporting Information), which lead to cancellation of the pathways involving CT states due to destructive interference. ${ }^{64} \mathrm{On}$ the other hand, addition of the DE states to the reduced model (see Fig. 4 (c)) results in a sizable population of $\left.\left.\right|^{1}\left(\mathrm{~T}_{1} \mathrm{~T}_{1}\right)\right\rangle$ of $\sim 25 \%$, which is, however, still significantly smaller than for the full model. From these results, we conclude that the participation of DE states that mix with the LE, ME, and CT states is necessary for efficient iSF in $o$-TIPSPm.

However, this condition is not sufficient and the process requires also the synergistic contribution of specific molecular vibrations (see Table S1 and Fig. S2 in Supporting Information). To illustrate this key point, we have simulated the photoinduced dynamics for a series of reduced vibronic model Hamiltonians that differ from the complete model in the number of vibrational modes included. The results show that among the 28 vibrational normal modes included in the simulation of Fig. 3 there is a subset of 8 modes that are fundamental for the iSF process. Figure 5 (a) depicts the time evolution of the population of the different diabatic states when the vibronic model Hamiltonian includes only these 8 modes. With this subset of vibrations, the $\left.\left.\right|^{1}\left(\mathrm{~T}_{1} \mathrm{~T}_{1}\right)\right\rangle$ state acquires a population of $\sim 40 \%$ at 
$300 \mathrm{fs}$, i.e. nearly two thirds of that obtained with the full model (see Fig. 3). Inspection of the normal modes included in the simulation (see Supporting Information) reveals that most of them are ring-breathing- and stretching-like modes involving the pentacene moities that exhibit frequencies in the range $0.1-0.2 \mathrm{eV}$, which are in line with the energy gaps between the electronic states considered in the model,therefore facilitating the process. ${ }^{31}$ Interestingly, and in agreement with previous work, ${ }^{16,22,65,66}$ one of the ring-breathing modes (with frequency $1350 \mathrm{~cm}^{-1}$ ) plays a key role. As shown in Fig. 5 (b) for a vibronic model Hamiltonian which includes only this normal mode, the coupling to this mode induces significant population of the $\left.\left.\right|^{1}\left(\mathrm{~T}_{1} \mathrm{~T}_{1}\right)\right\rangle$ for shorter times $(\sim 27 \%$ at $150 \mathrm{fs})$, very similar to the results found for the 8-mode model. For longer times, due to the reduced phase space of this model, recurrences set in and the dynamics is quite different. A cut of the potential energy surface along this mode (see Supporting Information for details) shows that the population transfer from the initially excited state to the $\left.\left.\right|^{1}\left(T_{1} T_{1}\right)\right\rangle$ state is facilitated by the enhancement of the coupling via the modulation of the energy gaps among the different adiabatic states and the changes in the degree of $\mathrm{CT}$ and $\mathrm{DE}$ character of the states upon distortion.

In summary, we have investigated the dynamics of intramolecular SF in a dimer consisting of two 6,13-bis(methylethynyl)pentacene chromophores covalently bonded to a phenylene spacer in ortho-conformation. We have shown that iSF takes place on an ultrafast subpicosecond timescale via a mediated mechanism. In the system considered, the virtual states that facilitate the population of the ME state in a superexchange-like way are both highlying CT and DE states, therefore providing a new mechanism to enhance iSF when the direct coupling is negligible and when the CT mediated coupling is small due to destructive interference. We have also shown that a significant population of the ME state is only obtained when electronic-vibrational coupling is included in the calculation, pointing out the instrumental role played by molecular vibrations in iSF. Among all the vibrational modes considered, fast population of the ME state is facilitated by a small number of high frequency modes mainly of ring-breathing- and stretching-like character, which decrease the energy gap 
between the CT and DE states and the LE and ME states, thereby maximizing the effective iSF coupling. These results suggest a new way of enhancing iSF by controlling the energetics of DE states.

\section{Acknowledgement}

The authors thank D. Guldi, J. Zirzlmeier, and D. Casanova for helpful and inspiring discussions and the developers of the Heidelberg MCTDH package for providing their code. Funding is gratefully acknowledged from the Emerging Fields Initiative "Singlet Fission" of the Friedrich-Alexander-Universität Erlangen-Nürnberg, the Deutsche Forschungsgemeinschaft (DFG CO-1627/1-1), the Regional Council for Industry and Employment of the Principality of Asturias, and the European Regional Development Fund (ERDF). Generous allocation of computing time by the computing center in Erlangen (RRZE) is gratefully acknowledged.

\section{Supporting Information Available}

Further details on the theoretical methods and list of the parameters used in the simulations. This material is available free of charge via the Internet at http://pubs.acs.org/.

\section{Notes}

The authors declare no competing financial interests.

\section{References}

(1) Shockley, W.; Queisser, H. J. Detailed Balance Limit of Efficiency of p-n Junction Solar Cells. J. Appl. Phys. 1961, 32, 510-519. 
(2) Schaller, R. D.; Klimov, V. I. High Efficiency Carrier Multiplication in PbSe Nanocrystals: Implications for Solar Energy Conversion. Phys. Rev. Lett. 2004, 92, 186601.

(3) Smith, M. B.; Michl, J. Singlet Fission. Chem. Rev. 2010, 110, 6891-6936.

(4) Nozik, A. J.; Ellingson, R. J.; Micic, O. I.; Blackburn, J. L.; Yu, P.; Murphy, J. E.; Beard, M. C.; Rumbles, G. Unique Approaches to Solar Photon Conversion Based on Semiconductor Nanostructures and Novel Molecular Chromophores; Dynamics of Electron Relaxation, Interfacial Charge Transfer, and Carrier Multiplication. Proc. 27th DOE Solar Photochem. Res. Conf. 2004; pp 63-66.

(5) Hanna, M. C.; Nozik, A. J. Solar Conversion Efficiency of Photovoltaic and Photoelectrolysis Cells with Carrier Multiplication Absorbers. J. Appl. Phys. 2006, 100, 074510.

(6) Smith, M. B.; Michl, J. Recent Advances in Singlet Fission. Ann. Rev. Phys. Chem. 2013, 64, 361-386.

(7) Burdett, J. J.; Bardeen, C. J. The Dynamics of Singlet Fission in Crystalline Tetracene and Covalent Analogs. Acc. Chem. Res. 2013, 46, 1312-1320.

(8) Ehrler, B.; Wilson, M. W. B.; Rao, A.; Friend, R. H.; Greenham, N. C. Singlet Exciton Fission-Sensitized Infrared Quantum Dot Solar Cells. Nano Lett. 2012, 12, 1053-1057.

(9) Congreve, D. N.; Lee, J.; Thompson, N. J.; Hontz, E.; Yost, S. R.; Reusswig, P. D.; Bahlke, M. E.; Reineke, S.; Van Voorhis, T.; Baldo, M. A. External Quantum Efficiency Above $100 \%$ in a Singlet-Exciton-Fission-Based Organic Photovoltaic Cell. Science 2013, 340, 334-337.

(10) Zimmerman, P. M.; Zhang, Z.; Musgrave, C. B. Singlet Fission in Pentacene through Multi-Exciton Quantum States. Nat. Chem. 2010, 2, 648-652.

(11) Zimmerman, P. M.; Musgrave, C. B.; Head-Gordon, M. A Correlated Electron View of Singlet Fission. Acc. Chem. Res. 2013, 46, 1339-1347. 
(12) Beljonne, D.; Yamagata, H.; Brédas, J. L.; Spano, F. C.; Olivier, Y. Charge-Transfer Excitations Steer the Davydov Splitting and Mediate Singlet Exciton Fission in Pentacene. Phys. Rev. Lett. 2013, 110, 226402.

(13) Feng, X.; Luzanov, A. V.; Krylov, A. I. Fission of Entangled Spins: An Electronic Structure Perspective. J. Phys. Chem. Lett. 2013, 4, 3845-3852.

(14) Chan, W.-L.; Berkelbach, T. C.; Provorse, M. R.; Monahan, N. R.; Tritsch, J. R.; Hybertsen, M. S.; Reichman, D. R.; Gao, J.; Zhu, X.-Y. The Quantum Coherent Mechanism for Singlet Fission: Experiment and Theory. Acc. Chem. Res. 2013, 46, $1321-1329$.

(15) Zeng, T.; Hoffmann, R.; Ananth, N. The Low-Lying Electronic States of Pentacene and Their Roles in Singlet Fission. J. Am. Chem. Soc. 2014, 136, 5755-5764.

(16) Casanova, D. Electronic Structure Study of Singlet Fission in Tetracene Derivatives. $J$. Chem. Theory Comput. 2014, 10, 324-334.

(17) Mirjani, F.; Renaud, N.; Gorczak, N.; Grozema, F. C. Theoretical Investigation of Singlet Fission in Molecular Dimers: The Role of Charge Transfer States and Quantum Interference. J. Phys. Chem. C 2014, 118, 14192-14199.

(18) Coto, P. B.; Sharifzadeh, S.; Neaton, J. B.; Thoss, M. Low-Lying Electronic Excited States of Pentacene Oligomers: A Comparative Electronic Structure Study in the Context of Singlet Fission. J. Chem. Theory Comput. 2015, 11, 147-156.

(19) Tayebjee, M. J. Y.; Sanders, S. N.; Kumarasamy, E.; Campos, L. M.; Sfeir, M. Y.; McCamey, D. R. Quintet Multiexciton Dynamics in Singlet Fission. Nat. Phys. 2016, $13,182-188$.

(20) Weiss, L. R.; Bayliss, S. L.; Kraffert, F.; Thorley, K. J.; Anthony, J. E.; Bittl, R.; 
Friend, R. H.; Rao, A.; Greenham, N. C.; Behrends, J. Strongly Exchange-Coupled Triplet Pairs in an Organic Semiconductor. Nat. Phys. 2016, 13, 176-181.

(21) Tempelaar, R.; Reichman, D. R. Vibronic Exciton Theory of Singlet Fission. I. Linear Absorption and the Anatomy of the Correlated Triplet Pair State. J. Chem. Phys. 2017, $146,174703$.

(22) Havenith, R. W.; de Gier, H. D.; Broer, R. Explorative Computational Study of the Singlet Fission Process. Mol. Phys. 2012, 110, 2445-2454.

(23) Berkelbach, T. C.; Hybertsen, M. S.; Reichman, D. R. Microscopic Theory of Singlet Exciton Fission. II. Application to Pentacene Dimers and the Role of Superexchange. J. Chem. Phys. 2013, 138, 114103.

(24) Vallett, P. J.; Snyder, J. L.; Damrauer, N. H. Tunable Electronic Coupling and Driving Force in Structurally Well-Defined Tetracene Dimers for Molecular Singlet Fission: A Computational Exploration Using Density Functional Theory. J. Phys. Chem. A 2013, $117,10824-10838$.

(25) Renaud, N.; Grozema, F. C. Intermolecular Vibrational Modes Speed Up Singlet Fission in Perylenediimide Crystals. J. Phys. Chem. Lett. 2015, 6, 360-365.

(26) Yang, C.-H.; Hsu, C.-P. First-Principle Characterization for Singlet Fission Couplings. J. Phys. Chem. Lett. 2015, 6, 1925-1929.

(27) Akimov, A. V.; Prezhdo, O. V. Nonadiabatic Dynamics of Charge Transfer and Singlet Fission at the Pentacene $/ \mathrm{C}_{60}$ Interface. J. Am. Chem. Soc. 2014, 136, 1599-1608.

(28) Tamura, H.; Huix-Rotllant, M.; Burghardt, I.; Olivier, Y.; Beljonne, D. First-Principles Quantum Dynamics of Singlet Fission: Coherent versus Thermally Activated Mechanisms Governed by Molecular $\pi$ Stacking. Phys. Rev. Lett. 2015, 115, 107401. 
(29) Zeng, T. Through-Linker Intramolecular Singlet Fission: General Mechanism and Designing Small Chromophores. J. Phys. Chem. Lett. 2016, 7, 4405-4412.

(30) Zheng, J.; Xie, Y.; Jiang, S.; Lan, Z. Ultrafast Nonadiabatic Dynamics of Singlet Fission: Quantum Dynamics with the Multilayer Multiconfigurational Time-Dependent Hartree (ML-MCTDH) Method. J. Phys. Chem. C 2016, 120, 1375-1389.

(31) Fujihashi, Y.; Chen, L.; Ishizaki, A.; Wang, J.; Zhao, Y. Effect of High-frequency Modes on Singlet Fission Dynamics. J. Chem. Phys. 2017, 146, 044101.

(32) Minami, T.; Ito, S.; Nakano, M. Theoretical Study of Singlet Fission in Oligorylenes. J. Phys. Chem. Lett. 2012, 3, 2719-2723.

(33) Akdag, A.; Havlas, Z.; Michl, J. Search for a Small Chromophore with Efficient Singlet Fission: Biradicaloid Heterocycles. J. Am. Chem. Soc. 2012, 134, 14624-14631.

(34) Minami, T.; Ito, S.; Nakano, M. Fundamental of Diradical-Character-Based Molecular Design for Singlet Fission. J. Phys. Chem. Lett. 2013, 4, 2133-2137.

(35) Renaud, N.; Sherratt, P. A.; Ratner, M. A. Mapping the Relation between Stacking Geometries and Singlet Fission Yield in a Class of Organic Crystals. J. Phys. Chem. Lett. 2013, 4, 1065-1069.

(36) Wang, L.; Olivier, Y.; Prezhdo, O. V.; Beljonne, D. Maximizing Singlet Fission by Intermolecular Packing. J. Phys. Chem. Lett. 2014, 5, 3345-3353.

(37) Feng, X.; Kolomeisky, A. B.; Krylov, A. I. Dissecting the Effect of Morphology on the Rates of Singlet Fission: Insights from Theory. J. Phys. Chem. C 2014, 118, 1960819617.

(38) Zeng, T.; Ananth, N.; Hoffmann, R. Seeking Small Molecules for Singlet Fission: A Heteroatom Substitution Strategy. J. Am. Chem. Soc. 2014, 136, 12638-12647. 
(39) Zeng, T.; Goel, P. Design of Small Intramolecular Singlet Fission Chromophores: An Azaborine Candidate and General Small Size Effects. J. Phys. Chem. Lett. 2016, 7, $1351-1358$.

(40) Müller, A. M.; Avlasevich, Y. S.; Müllen, K.; Bardeen, C. J. Evidence for Exciton Fission and Fusion in a Covalently Linked Tetracene Dimer. Chem. Phys. Lett. 2006, 421, 518-522.

(41) Müller, A. M.; Avlasevich, Y. S.; Schoeller, W. W.; Müllen, K.; Bardeen, C. J. Exciton Fission and Fusion in Bis(tetracene) Molecules with Different Covalent Linker Structures. J. Am. Chem. Soc. 2007, 129, 14240-14250.

(42) Busby, E.; Xia, J.; Wu, Q.; Low, J. Z.; Song, R.; Miller, J. R.; Zhu, X.-Y.; Campos, L.; Sfeir, M. Y. A Design Strategy for Intramolecular Singlet Fission Mediated by ChargeTransfer States in Donor-Acceptor Organic Materials. Nat. Mater. 2015, 14, 426-433.

(43) Zirzlmeier, J.; Lehnherr, D.; Coto, P. B.; Chernick, E. T.; Casillas, R.; Basel, B. S.; Thoss, M.; Tykwinski, R. R.; Guldi, D. M. Singlet Fission in Pentacene Dimers. Proc. Natl. Acad. Sci. U. S. A. 2015, 112, 5325-5330.

(44) Zirzlmeier, J.; Casillas, R.; Reddy, S. R.; Coto, P. B.; Lehnherr, D.; Chernick, E. T.; Papadopoulos, I.; Thoss, M.; Tykwinski, R. R.; Guldi, D. M. Solution-Based Intramolecular Singlet Fission in Cross-Conjugated Pentacene Dimers. Nanoscale 2016, 8, 1011310123.

(45) Basel, B. S.; Zirzlmeier, J.; Hetzer, C.; Phelan, B. T.; Krzyaniak, M. D.; Reddy, S. R.; Coto, P. B.; Horwitz, N. E.; Young, R. M.; White, F. J. et al. Unified Model for Singlet Fission within a Non-conjugated Covalent Pentacene Dimer. Nat. Commun. 2017, 8, 15171.

(46) Kumarasamy, E.; Sanders, S. N.; Tayebjee, M. J. Y.; Asadpoordarvish, A.; Hele, T. J. H.; Fuemmeler, E. G.; Pun, A. B.; Yablon, L. M.; Low, J. Z.; Paley, D. W. et al. 
Tuning Singlet Fission in $\pi$-Bridge- $\pi$ Chromophores. J. Am. Chem. Soc. 2017, 139, $12488-12494$.

(47) Trinh, M. T.; Pinkard, A.; Pun, A. B.; Sanders, S. N.; Kumarasamy, E.; Sfeir, M. Y.; Campos, L. M.; Roy, X.; Zhu, X.-Y. Distinct Properties of the Triplet Pair State from Singlet Fission. Sci. Adv. 2017, 3, e1700241.

(48) Yang, L.; Tabachnyk, M.; Bayliss, S. L.; Böhm, M. L.; Broch, K.; Greenham, N. C.; Friend, R. H.; Ehrler, B. Solution-Processable Singlet Fission Photovoltaic Devices. Nano Lett. 2015, 15, 354-358.

(49) Musser, A. J.; Liebel, M.; Schnedermann, C.; Wende, T.; Kehoe, T. B.; Rao, A.; Kukura, P. Evidence for Conical Intersection Dynamics Mediating Ultrafast Singlet Exciton Fission. Nat. Phys. 2015, 11, 352-357.

(50) Bakulin, A. A.; Morgan, S. E.; Kehoe, T. B.; Wilson, M. W. B.; Chin, A. W.; Zigmantas, D.; Egorova, D.; Rao, A. Real-Time Observation of Multiexcitonic States in Ultrafast Singlet Fission using Coherent 2D Electronic Spectroscopy. Nat. Chem. 2015, $8,16-23$.

(51) Meyer, H.-D.; Manthe, U.; Cederbaum, L. The Multi-Configurational Time-Dependent Hartree Approach. Chem. Phys. Lett. 1990, 165, 73-78.

(52) Beck, M.; Jäckle, A.; Worth, G.; Meyer, H.-D. The Multiconfiguration Time-Dependent Hartree (MCTDH) Method: a Highly Efficient Algorithm for Propagating Wavepackets. Phys. Rep. 2000, 324, 1-105.

(53) Wang, H.; Thoss, M. Multilayer Formulation of the Multiconfiguration Time-dependent Hartree Theory. J. Chem. Phys. 2003, 119, 1289-1299.

(54) Manthe, U. A Multilayer Multiconfigurational Time-dependent Hartree Approach for 
Quantum Dynamics on General Potential Energy Surfaces. J. Chem. Phys. 2008, 128, 164116.

(55) Meyer, H.-D., Gatti, F., Worth, G. A., Eds. Multidimensional Quantum Dynamics; Wiley-VCH Verlag GmbH \& Co.: Weinheim, 2009.

(56) Vendrell, O.; Meyer, H.-D. Multilayer Multiconfiguration Time-dependent Hartree Method: Implementation and Applications to a Henon-Heiles Hamiltonian and to Pyrazine. J. Chem. Phys. 2011, 134, 044135.

(57) Wang, H. Multilayer Multiconfiguration Time-Dependent Hartree Theory. J. Phys. Chem. A 2015, 119, 7951-7965.

(58) Wang, H.; Thoss, M. Employing an Interaction Picture to Remove Artificial Correlations in Multilayer Multiconfiguration Time-dependent Hartree Simulations. J. Chem. Phys. 2016, 145, 164105.

(59) Köppel, H.; Domcke, W.; Cederbaum, L. S. Multimode Molecular Dynamics Beyond the Born-Oppenheimer Approximation. Adv. Chem. Phys. 1984, 57, 59-246.

(60) Nakamura, H.; Truhlar, D. G. The Direct Calculation of Diabatic States based on Configurational Uniformity. J. Chem. Phys. 2001, 115, 10353-10372.

(61) Nakamura, H.; Truhlar, D. G. Direct Diabatization of Electronic States by the Fourfold Way. II. Dynamical Correlation and Rearrangement Processes. J. Chem. Phys. 2002, $117,5576-5593$.

(62) Granovsky, A. A. Extended Multi-Configuration Quasi-Degenerate Perturbation Theory: The New Approach to Multi-State Multi-Reference Perturbation Theory. J. Chem. Phys. 2011, 134, 214113.

(63) Dunning, T. H.; Hay, P. J. In Methods of Electronic Structure Theory; Schaefer III, H. F., Ed.; Springer US: New York, 2013; Chapter 1, pp 1-27. 
(64) Castellanos, M. A.; Huo, P. Enhancing Singlet Fission Dynamics by Suppressing Destructive Interference between Charge-Transfer Pathways. J. Phys. Chem. Lett. 2017, 8, 2480-2488.

(65) Yamagata, H.; Norton, J.; Hontz, E.; Olivier, Y.; Beljonne, D.; Brédas, J. L.; Silbey, R. J.; Spano, F. C. The Nature of Singlet Excitons in Oligoacene Molecular Crystals. J. Chem. Phys. 2011, 134, 204703.

(66) Fuemmeler, E. G.; Sanders, S. N.; Pun, A. B.; Kumarasamy, E.; Zeng, T.; Miyata, K.; Steigerwald, M. L.; Zhu, X.-Y.; Sfeir, M. Y.; Campos, L. M. et al. A Direct Mechanism of Ultrafast Intramolecular Singlet Fission in Pentacene Dimers. ACS Cent. Sci. 2016, 2, 316-324. 